\title{
Medical isotope collection from ISAC targets
}

Peter Kunz ${ }^{1,2, *}$, Corina Andreoiu ${ }^{2}$, Victoria Brown ${ }^{1}$, Marla Cervantes $^{1}$, Julia Even ${ }^{5}$, Fatima H. Garcia ${ }^{2}$, Alexander Gottberg $^{1}$, Jens Lassen ${ }^{1}$, Valery Radchenko ${ }^{1,4}$, Caterina F. Ramogida ${ }^{1,2}$, Andrew K. H. Robertson ${ }^{1,3}$, Paul Schaffer ${ }^{1,2}$, and Rozhannaa Sothilingam ${ }^{1}$

${ }^{1}$ TRIUMF, 4004 Wesbrook Mall, Vancouver, BC V6T 2A3, Canada

${ }^{2}$ Department of Chemistry, Simon Fraser University, Burnaby, BC V5A 1S6, Canada

${ }^{3}$ Physics and Astronomy, University of British Columbia, Vancouver, BC, V6T 1Z4, Canada

${ }^{4}$ Department of Chemistry, University of British Columbia, Vancouver, BC, V6T 1Z1, Canada

${ }^{5}$ University of Groningen, Zernikelaan 25, 9747 AA Groningen, Netherlands

\begin{abstract}
The ISAC facility (Isotope Separation and Acceleration) at TRIUMF has recently started to provide isotopes for pre-clinical nuclear medicine studies. By irradiating ISOL (Isotope Separation OnLine) targets with a $480 \mathrm{MeV}$ proton beam from the TRIUMF H- cyclotron, the facility can deliver a large variety of radioactive isotope beams (RIB) for research in the fields of nuclear astrophysics, nuclear structure and material science with half-lives down to a few milliseconds via an electrostatic beamline network. For the collection of medical isotopes, typically with half-lives in the range of hours or days, we have developed a compact apparatus for the implantation of mass-separated RIB on a target disc at energies between 20-55 keV. In this paper, we also discuss two different retrieval methods of the implanted activity from the implantation target: by chemical etching of the target surface and by recoil collection of implanted alpha emitters.
\end{abstract}

\section{Introduction}

The ISAC facility features two target stations for the irradiation of ISOL-type thick targets with up to $100 \mu \mathrm{A}$ of a $480 \mathrm{MeV}$ proton beam from the TRIUMF H- cyclotron. Using a variety of refractory target materials (metal foils, composite ceramic carbides, oxides) a large number of radioactive isotope beams (RIB) can be produced by spallation, fragmentation or fission from elements such as $\mathrm{C}$, $\mathrm{Si}, \mathrm{Ti}, \mathrm{Ni}, \mathrm{Nb}, \mathrm{Ta}$, Th and $\mathrm{U}$. Only sufficiently volatile isotopes in atomic or molecular form for which the average release time doesn't exceed their half-life too much can be released, ionized, mass-separated and distributed through the ISAC electrostatic beamline network. Therefore, to accommodate a fast and efficient release, ISAC targets are generally operated at temperatures as high as permissible by the properties of the target material - mainly vapor pressure, thermal conductivity, sintering behavior and chemical stability. Operating temperatures range from $1100{ }^{\circ} \mathrm{C}$ for nickel oxide targets to $2300{ }^{\circ} \mathrm{C}$ for tantalum metal foil targets [1]. The facility is mainly used for research in the fields of nuclear astrophysics, nuclear structure and material science [2]. In addition to these applications, the facility is now also producing radioisotopes for pre-clinical nuclear medicine research. Some of those isotopes are of interest for imaging or therapeutic applications or both (theranostic isotopes). They generally have half-lives in the range of hours or days and can be produced in sufficient quantities for pre-clinical research from

*e-mail: pkunz@triumf.ca a variety of target materials. The current status of verified RIB yields is available for ISAC users in the ISAC Yield Database [3].

This paper is providing an overview of of isotopes that have already been collected as well as potential candidates for future applications related to nuclear medicine research. In particular, the collection of the heavy, alpha emitting isotope ${ }^{225} \mathrm{Ac}$ and its precursor ${ }^{225} \mathrm{Ra}$ are discussed. ${ }^{225} \mathrm{Ac}$ [4] and its decay product ${ }^{213} \mathrm{Bi}$ are promising candidates for targeted alpha therapy (TAT). Furthermore, methods to transfer the collected activity from the implantation target have been investigated. Sample preparation methods such as chemical etching of implantation targets and the recoil collection of ${ }^{213} \mathrm{Bi}$ from the ${ }^{225} \mathrm{Ra} / \mathrm{Ac}$ decay chain are discussed.

\section{Experimental}

The two target stations at the ISAC facility are operated alternately. The $480 \mathrm{MeV}$ proton beam can be directed at either one of them where it impinges on a production target with a beam current of up to $100 \mu \mathrm{A}$ with a maximum achievable beam power of $48 \mathrm{~kW}$. In order to accommodate the full capacity of the cyclotron, high-power tantalum target containers [5] have been developed. The addition of cooling fins increases the emissivity to $92 \%$ and allows to dissipate up to $25 \mathrm{~kW}$ of beam power. This is sufficient since power deposition in the target material is usually less than $50 \%$ of the maximum beam power. Thickness, density, thermal conductivity, vapor pressure are fac- 


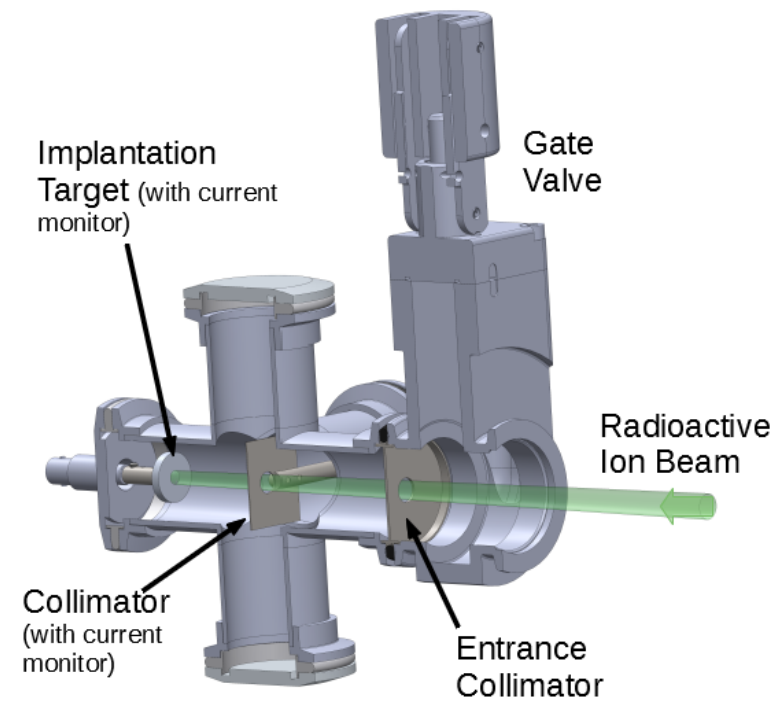

Fig. 1. Section view of the ISAC Implantation chamber. The RIB is focused though a set of collimators on the implantation target in the back of the chamber.

tors that determine the maximum feasible beam power on target. The extraction of a beam from the ion source is only efficient under high-vacuum conditions $\left(<10^{-5} \mathrm{mbar}\right)[6]$. The vapor pressure depends strongly on the target temperature which depends on power deposition and the efficiency of power dissipation, governed by the thermal conductivity of the target material and the emissivity of the target container. The resulting target temperature limitations can be detrimental to the efficient and fast release of isotopes through diffusion and effusion which benefits from high operating temperatures. The best balance between these two opposing requirements can be achieved by using refractory materials with high thermal stability, low vapor pressure and good thermal conductivity. Another aspect considering target material properties are diffusion times. Slow diffusion reduces the release efficiency of short-lived isotopes. This effect can be mitigated by using high-porosity materials. For example, the average bulk density of the uranium carbide used for ISAC targets is typically around $3.3 \mathrm{~g} / \mathrm{cm}^{3}$ [7], about $1 / 3$ of the theoretical density of $\mathrm{UC}_{2}$. In comparison to bulk $\mathrm{UC}_{2}$, the thermal conductivity of this porous material is reduced by a factor of 6 . In order to still achieve sufficient power dissipation, composite ceramic target materials [8], consisting of disks made of a 100-250 $\mu$ m thick carbide layer on a $130 \mu \mathrm{m}$ graphite backing foil have been developed. This configuration increases the o verall thermal conductivity significantly due to the relatively high conductivity of the graphite backing foils. For low-thermal conductivity oxide materials $\left(\mathrm{NiO}, \mathrm{UO}_{2}\right) 25 \mu \mathrm{m}$ thick niobium metal backing foils serve as thermal conductivity booster.

The production rate for a specific isotope is the product of production cross section, proton beam intensity and target thickness. The actual isotope beam intensity that arrives at the experiment also depends on the release efficiency form the target, the ionization efficiency and the transport efficiency of the ion beam through the ISAC electrostatic beamline network. Beam transport includes the extraction and acceleration of ions from the ion source to energies between 10-55 keV, subsequent mass separation through a low resolution pre-separator, a high-resolution mass separator $(\mathrm{m} / \Delta \mathrm{m}=2000)$.

For the ionization process a variety of ion source options can be utilized [9]. Which ionization method is most suitable, depends strongly on the ionization potential of the atoms or molecules released from the target. The ion beams discussed in this paper were ionized with a surface ion source or by resonant laser ionization.

For the production of heavy isotopes for targeted alpha therapy research, composite ceramic uranium carbide targets [7] are currently used. They have typical thicknesses between 0.05 and $0.1 \mathrm{~mol} \mathrm{U} / \mathrm{cm}^{2}$ and are irradiated with a proton beam of max. $10 \mu \mathrm{A}$ for up to $5000 \mu \mathrm{Ah}$ (current operation license limits) at an operating temperature of approximately $1950{ }^{\circ} \mathrm{C}$. Neutron-deficient isotopes for medical applications in the lanthanide region are efficiently produced from tantalum metal foil targets, which are filled with several hundred $25 \mu \mathrm{m}$ thick Ta foil disks up to a total thickness of $0.14 \mathrm{~mol} / \mathrm{cm}^{2}$. They are operated at up to $70 \mu \mathrm{A}$ proton beam current and a target core temperature of $2300^{\circ} \mathrm{C}$ [6].

\subsection{ISAC Implantation Station (IIS)}

The IIS is located a few meters behind the ISAC highresolution mass separator on a short branch of the electrostatic beamline. It is divided in two sections. Section 1 contains ion beam optics for beam positioning, focusing or rastering. It ends at a gate valve with a pumping port on it's downstream side which provides vacuum for the ion collection chamber (section 2). This chamber can be custom-build for specific applications and is attached to the gate valve by the user.

The design of the ion collection chamber used for the examples presented in this paper is shown in figure 1. It is made of a standard KF40 6-way cross. Attached are vent and sampling valves to test for airborne contamination, a vacuum gauge, a collimator with current readout for beam centering and the implantation target mounted on an electrical feedthrough to allow current monitoring during the implantation process. In principle the target can have any form and composition. The only condition is that it must be electrically conductive to avoid charging and enable a current readback. For the experiments in this paper we used a standard aluminium SEM sample holder ( $\varnothing 25 \mathrm{~mm})$ with a machined-in $2 \mathrm{~mm}$ deep cut-out ( $\varnothing 16 \mathrm{~mm}$ ), see figure 2. The cut-out is required for the etching process described in 2.2. A manual KF40 gate valve serves as an interface to section 1. Once an implantation is finished the gate valves in both sections are closed and the volume between them is vented. Now, the ion collection chamber can be detached and - still under vacuum - be transferred to the radiochemistry laboratory. This way, the collected activity can be considered a closed source, which is important particularly for alpha emitters where the spread of contamination through recoil emission can be a problem. 

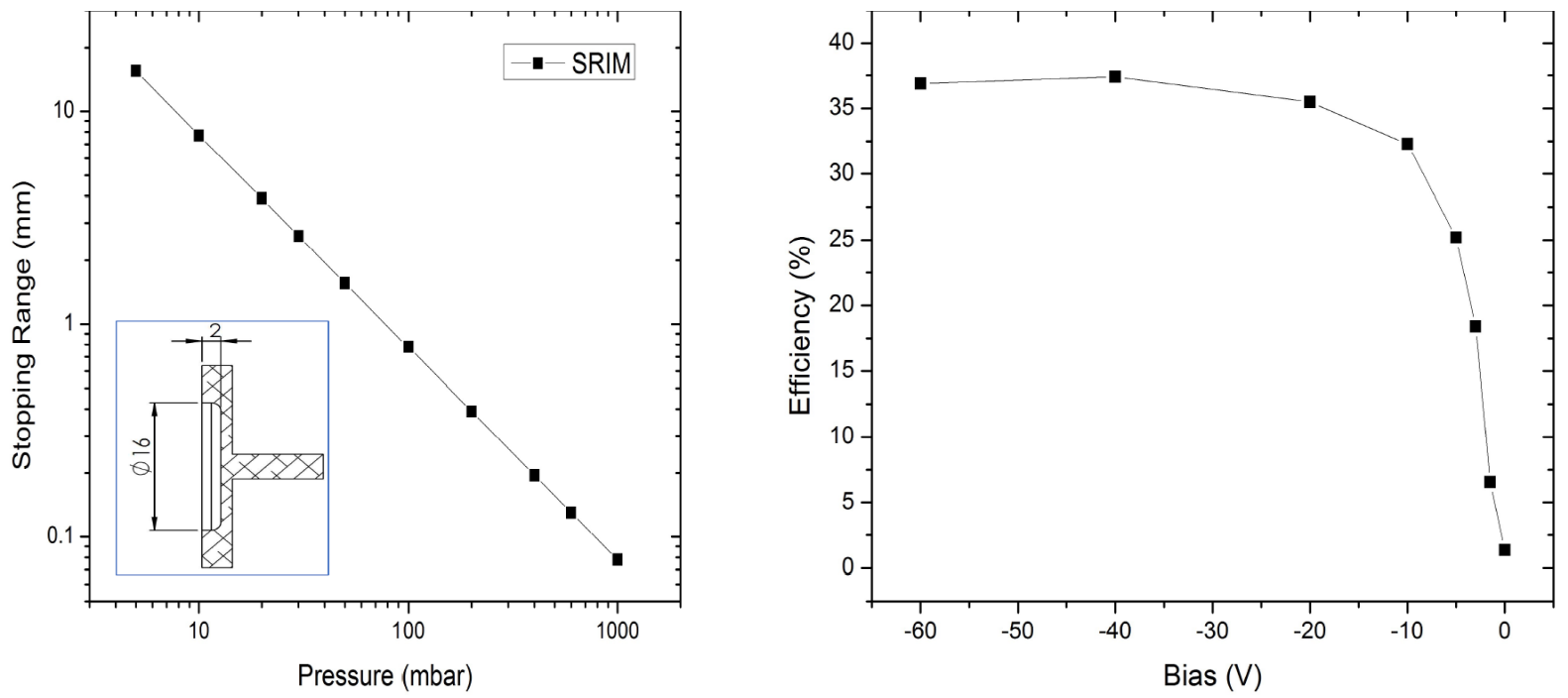

Fig. 2. ${ }^{225} \mathrm{Ac}$ recoil collection depends on the stopping range of approx. $100 \mathrm{keV}$ alpha decay recoils (left) and the bias between the implantation target (insert, dimensions in $\mathrm{mm}$ ) and an identical ion catcher target. The measured ${ }^{213} \mathrm{Bi}$ collection efficiency (right) at normal air pressure for a configuration in which the catcher target was pressed against the implantation target, separated by a thin insulator. A negative bias was applied to the catcher.

Once in the lab, the chamber can be vented and the implantation target can be retrieved for further processing.

\subsection{Retrieval of activity from IIS targets}

We have explored 2 methods to retrieve radioactive isotopes from the implantation target: chemical etching of the target surface (2.2.1) and recoil collection from implanted alpha emitters (2.2.2)

\subsubsection{Chemical etching}

The implantation depth of a heavy ${ }^{225} \mathrm{Ac} / \mathrm{Ra}$ ion beam at a typical ISAC extraction energy of $30 \mathrm{keV}$ in aluminium is about $20 \mathrm{~nm}$ [10]. Therefore, the transfer of the implanted isotopes into an aqueous solution requires the removal of at least $20 \mathrm{~nm}$ of the implantation target surface.

To achieve this, $0.1 \mathrm{ml}$ of $0.1 \mathrm{~N} \mathrm{HCl}$ is filled into the implantation target cut-out and then gently evaporated on a hot plate. Then the target is cooled down and a thin layer of $\mathrm{AlCl}_{3}$, that has formed during the evaporation, is dissolved with $0.1 \mathrm{ml} 0.1 \mathrm{~N} \mathrm{HCl}$. This solution is picked up with a micropipette and transferred to a vial. This etchrinse procedure is repeated several times. 3 iterations already remove $>95 \%$ of the implanted activity, leaving it in a small volume of acidic solution which can be used for further radiochemical experiments.

\subsubsection{Recoil collection}

Isotopes like ${ }^{223-225} \mathrm{Ra}$ and ${ }^{225} \mathrm{Ac}$ decay through relatively long chains, including multiple alpha decays. The recoil energy transferred during an alpha decay to the residual nucleus is around $100 \mathrm{keV}$, about 3 times larger than the typical ion beam implantation energy at ISAC. Therefore, the alpha decay recoils have sufficient kinetic energy to escape as long as the recoil vector points out of the target. For a thick implantation target, as was used in these experiments, this leads to a theoretical release efficiency of less than $50 \%$ for a single alpha decay. However, the collection of ${ }^{213} \mathrm{Bi}$ from a ${ }^{225} \mathrm{Ac}$ source involves 3 alpha decays in the chain. The decays of ${ }^{221} \mathrm{Fr}$ and ${ }^{217}$ At contribute to the overall release efficiency, increasing it to $60 \%$ at an implantation energy of $30 \mathrm{keV}$. This result was confirmed with a GEANT4 [11] recoil track simulation.

Using a ${ }^{225} \mathrm{Ac} / \mathrm{Ra}$ target as shown in figure $2,{ }^{213} \mathrm{Bi}$ recoils were accumulated by placing an identical collection target, separated by a thin insulator, directly on top. The collection was ended when the activity of ${ }^{213} \mathrm{Bi}\left(\mathrm{T}_{1 / 2}=\right.$ $45.59 \mathrm{~min}$ ) had reached saturation. Figure 2 (right) shows that a negative bias of $>20 \mathrm{~V}$ lead to a collection efficiency for ${ }^{213} \mathrm{Bi}$ of $\sim 35 \%$.

The stopping range of recoils in air under normal pressure is less than $0.1 \mathrm{~mm}$, see figure 2 (left). Aligning the stopping range more accurately with the distance between source and collector by reducing the pressure to 50 mbar and using argon as a buffer gas increased the collection efficiency to $47 \%$. However, for applications such as chelation studies a $35 \%$ efficiency was sufficient and sample collection under normal air pressure much more convenient.

\section{Conclusion}

All measured yield rates from ISAC targets are compiled in Ref. [3]. From these values, activities can be extrapolated either until saturation is reached or to a maximum 24 hour implantation period. This is a realistic time frame, considering that radioactive ion beam time is usually allocated for several experiments with one ISAC target. Within this period, a ${ }^{225}$ Ac beam $\left(\mathrm{T}_{1 / 2}=9.92 \mathrm{~d}\right)$ at a rate of $1.3 \cdot 10^{8}$ ions/s from a uranium carbide target / laser 
Table 1. Selected isotopes for potential medical applications from ISAC targets. The table lists primary collection beam, target material, ion source and established yields [3] and the activity that can be accumulated within a typical $24 \mathrm{~h}$ beam collection period.

\begin{tabular}{|c|c|c|c|c|c|c|c|}
\hline Isotope & Half-life & Application & $\begin{array}{l}\text { Primary } \\
\text { beam }\end{array}$ & $\begin{array}{l}\text { Target } \\
\text { material }\end{array}$ & $\begin{array}{l}\text { Ion } \\
\text { source }\end{array}$ & $\begin{array}{l}\text { Measured yield } \\
\text { [ions/s] }\end{array}$ & $\begin{array}{l}\text { Accumulated } \\
\text { 24h Activity [Bq] }\end{array}$ \\
\hline $225 \mathrm{Ac}$ & $9.92 \mathrm{~d}$ & TAT $^{a}$ & $225 \mathrm{Ac}$ & $\mathrm{UC}$ & $\mathrm{LIS}^{f f}$ & $1.30 \mathrm{E}+08$ & $8.60 \mathrm{E}+06$ \\
\hline $225 \mathrm{Ac}$ & $9.92 \mathrm{~d}$ & $\mathrm{TAT}^{a}$ & $225 \mathrm{Ra}$ & $\mathrm{UC}$ & SIS $^{g g}$ & $1.70 \mathrm{E}+08$ & $3.40 \mathrm{E}+06$ \\
\hline $224 \mathrm{Ra}$ & $3.66 \mathrm{~d}$ & TAT $^{a}$ & $224 \mathrm{Ra}$ & $\mathrm{UC}$ & $\mathrm{SIS}^{g}$ & $1.60 \mathrm{E}+09$ & $2.70 \mathrm{E}+08$ \\
\hline 223Ra & $11.43 \mathrm{~d}$ & TAT $^{a}$ & 223Ra & ThO & $\mathrm{SIS}^{g}$ & $6.90 \mathrm{E}+08$ & $4.00 \mathrm{E}+07$ \\
\hline 213Bi & $45.6 \mathrm{~m}$ & TAT $^{a}$ & $225 \mathrm{Ac}$ & $\mathrm{UC}$ & $\mathrm{SIS}^{g}$ & $6.70 \mathrm{E}+07$ & $2.25 \mathrm{E}+06$ \\
\hline $212 \mathrm{~Pb}$ & $10.64 \mathrm{~h}$ & TAT $^{a}$ & $224 \mathrm{Ra}$ & $\mathrm{UC}$ & $\mathrm{SIS}^{g}$ & $1.60 \mathrm{E}+09$ & $1.00 \mathrm{E}+08$ \\
\hline 212Bi & $60.6 \mathrm{~m}$ & TAT $^{a}$ & $224 \mathrm{Ra}$ & $\mathrm{UC}$ & SIS $^{g}$ & $1.60 \mathrm{E}+09$ & $7.80 \mathrm{E}+07$ \\
\hline $211 \mathrm{At}$ & $7.22 \mathrm{~h}$ & TAT $^{a}$ & $211 R n$ & UO & FEBIAD $^{h}$ & $1.00 \mathrm{E}+08$ & $2.40 \mathrm{E}+07$ \\
\hline $211 \mathrm{At}$ & $7.22 \mathrm{~h}$ & TAT $^{a}$ & $211 \mathrm{At}$ & UO & FEBIAD $^{h}$ & $8.40 \mathrm{E}+07$ & $7.40 \mathrm{E}+07$ \\
\hline $211 \mathrm{At}$ & $7.22 \mathrm{~h}$ & TAT $^{a}$ & $211 \mathrm{Fr}$ & $\mathrm{UC}$ & SIS $^{g}$ & $1.90 \mathrm{E}+09$ & $4.30 \mathrm{E}+07$ \\
\hline 209At & $5.4 \mathrm{~h}$ & $\mathrm{SPECT}^{b}$ & $213 \mathrm{Fr}$ & $\mathrm{UC}$ & $\mathrm{SIS}^{g}$ & $1.80 \mathrm{E}+09$ & $8.50 \mathrm{E}+08$ \\
\hline 209At & $5.4 \mathrm{~h}$ & $\mathrm{SPECT}^{b}$ & 209At & ThO & $\operatorname{LIS}^{f}$ & $1.90 \mathrm{E}+08$ & $1.70 \mathrm{E}+08$ \\
\hline $177 \mathrm{Lu}$ & $6.65 \mathrm{~d}$ & $\mathrm{BT}^{c}$ & $177 \mathrm{Lu}$ & $\mathrm{Ta}$ & $\operatorname{LIS}^{f}$ & $6.50 \mathrm{E}+08$ & $6.40 \mathrm{E}+07$ \\
\hline $169 \mathrm{Yb}$ & $32.02 \mathrm{~d}$ & SPECT $^{b}$ & $169 \mathrm{Yb}$ & $\mathrm{Ta}$ & $\operatorname{LIS}^{f}$ & $5.10 \mathrm{E}+10$ & $1.00 \mathrm{E}+09$ \\
\hline $166 \mathrm{Yb}$ & $2.36 \mathrm{~d}$ & $\mathrm{AT}^{d}$ & $166 \mathrm{Yb}$ & $\mathrm{Ta}$ & $\operatorname{LIS}^{f}$ & $1.50 \mathrm{E}+11$ & $3.70 \mathrm{E}+10$ \\
\hline $165 \mathrm{Er}$ & $10.3 \mathrm{~h}$ & $\mathrm{AT}^{d}$ & $165 \mathrm{Yb} / 165 \mathrm{Tm}$ & Ta & LIS $^{f}$ & $9.32 \mathrm{E}+10$ & $3.90 \mathrm{E}+10$ \\
\hline $161 \mathrm{Ho}$ & $2.5 \mathrm{~h}$ & $\mathrm{AT}^{d}$ & $161 \mathrm{Ho}$ & $\mathrm{Ta}$ & LIS $^{f}$ & $9.96 \mathrm{E}+09$ & $9.30 \mathrm{E}+09$ \\
\hline $161 \mathrm{Ho}$ & $2.5 \mathrm{~h}$ & $\mathrm{AT}^{d}$ & $161 \mathrm{Er}$ & $\mathrm{Ta}$ & $\operatorname{LIS}^{f}$ & $1.03 \mathrm{E}+10$ & $3.90 \mathrm{E}+09$ \\
\hline $149 \mathrm{~Tb}$ & $4.12 \mathrm{~h}$ & $\mathrm{TAT}^{a} / \mathrm{SPECT}^{b}$ & $149 \mathrm{~Tb}$ & $\mathrm{Ta}$ & $\mathrm{SIS}^{g}$ & $5.78 \mathrm{E}+08$ & $5.75 \mathrm{E}+08$ \\
\hline $140 \mathrm{Nd}$ & $3.37 \mathrm{~d}$ & $\mathrm{PET}^{e} / \mathrm{AT}^{c}$ & $140 \mathrm{Sm}$ & $\mathrm{Ta}$ & LIS $^{f}$ & $9.70 \mathrm{E}+08$ & $1.70 \mathrm{E}+08$ \\
\hline 124I & $4.18 \mathrm{~d}$ & $\mathrm{PET}^{e}$ & $124 \mathrm{I}$ & UO & FEBIAD $^{h}$ & $2.20 \mathrm{E}+08$ & $3.20 \mathrm{E}+07$ \\
\hline $123 \mathrm{I}$ & $13.22 \mathrm{~h}$ & $\mathrm{PET}^{e}$ & $123 \mathrm{I}$ & UO & FEBIAD $^{h}$ & $3.80 \mathrm{E}+07$ & $2.60 \mathrm{E}+07$ \\
\hline $82 \mathrm{Sr}$ & $1.35 \mathrm{~d}$ & $\mathrm{PET}^{e}$ & $82 \mathrm{Sr}$ & $\mathrm{Nb}$ & SIS $^{g}$ & $1.00 \mathrm{E}+10$ & $2.10 \mathrm{E}+08$ \\
\hline $77 \mathrm{Br}$ & $57.0 \mathrm{~h}$ & Labeling & $77 \mathrm{Rb}$ & $\mathrm{Nb}$ & SIS $^{g}$ & $1.60 \mathrm{E}+09$ & $3.70 \mathrm{E}+08$ \\
\hline $67 \mathrm{Ga}$ & $78.28 \mathrm{~h}$ & Imaging & $67 \mathrm{Ga}$ & $\mathrm{ZrC}$ & $\operatorname{LIS}^{f}$ & $8.10 \mathrm{E}+09$ & $1.50 \mathrm{E}+09$ \\
\hline $67 \mathrm{Cu}$ & $2.58 \mathrm{~d}$ & $\mathrm{BT}^{c}$ & $67 \mathrm{Cu}$ & Тa & $\operatorname{LIS}^{f}$ & $1.40 \mathrm{E}+08$ & $3.10 \mathrm{E}+07$ \\
\hline $64 \mathrm{Cu}$ & $12.70 \mathrm{~h}$ & $\mathrm{PET}^{e} /$ therapy & $64 \mathrm{Cu}$ & $\mathrm{Nb}$ & $\operatorname{LIS}^{f}$ & $3.20 \mathrm{E}+09$ & $2.20 \mathrm{E}+09$ \\
\hline $61 \mathrm{Cu}$ & $3.33 \mathrm{~h}$ & $\mathrm{PET}^{e}$ & $61 \mathrm{Cu}$ & $\mathrm{Nb}$ & $\operatorname{LIS}^{f}$ & $7.80 \mathrm{E}+08$ & $7.50 \mathrm{E}+08$ \\
\hline
\end{tabular}

${ }^{a}$ targeted alpha therapy, ${ }^{b}$ single-photon emission computed tomography, ${ }^{c}$ beta therapy, ${ }^{d}$ Auger therapy, ${ }^{e}$ positron emission tomography, ${ }^{f}$ laser ion source,,${ }^{g}$ surface ion source, ${ }^{h}$ forced electron beam induced arc discharge ion source

ion source combination accumulates an activity of up to $8.6 \cdot 10^{6} \mathrm{~Bq}$. The accompanying ${ }^{225} \mathrm{Ra}\left(\mathrm{T}_{1 / 2}=14.8 \mathrm{~d}\right)$ beam is collected simultaneously. At a rate of $1.7 \cdot 10^{8}$ ions/s from a uranium carbide target / surface ion source combination a total of $1.4 \cdot 10^{13}$ atoms $\left(7.5 \cdot 10^{6} \mathrm{~Bq}\right)$ can be collected in 24 hours. ${ }^{225} \mathrm{Ra}$ decays via $\beta$-decay into ${ }^{225} \mathrm{Ac}$, reaching a maximum activity of $3.4 \cdot 10^{6} \mathrm{~Bq}^{225} \mathrm{Ac}$ approximately 18 days after the implantation.

The etching method (section 2.2.1) was used to extract Ac and $\mathrm{Ra}$ isotopes. Then the radium was separated from the actinium by well established extraction chromatography methods [12]. The remaining ${ }^{225} \mathrm{Ra}$ can be recycled and used as an ${ }^{225}$ Ac generator. This has been demonstrated as part of the development of Multi-isotope SPECT imaging of the ${ }^{225}$ Ac decay chain by Robertson et. al. [13]. The collection of ${ }^{213} \mathrm{Bi}$ via recoil transfer was employed during chelation studies. In this case the collected activities were significantly lower, in the $\mathrm{kBq}$ range, but still sufficient for this kind of experiment. The recoil transfer from the low energy beta decay of ${ }^{225} \mathrm{Ra}$ is insignificant compared to the implantation energy and, therefore, stays in the implantation target, while it's decay product ${ }^{225} \mathrm{Ac}$ can escape, thus, extending the lifetime of the ${ }^{213} \mathrm{Bi}$ source significantly.

With the example of ${ }^{225} \mathrm{Ac} / \mathrm{Ra}$ beams, it has been demonstrated that the ISAC facility at TRIUMF has the capability of providing pure isotope samples for pre-clinical nuclear medicine research [14]. Infrastructure for the ISAC Implantation Station and methods for production and retrieval of medical isotopes have been established. Similar implantation procedures have also been applied to the production of 209/211At via the implantation of ${ }^{211 / 213}$ Fr beams for At-based $\alpha$-therapy research [15][16]. In principle, the methods described above are applicable for any isotope beam from ISAC targets.

Table 1 lists the established yield rates (Ref. [3]) of isotopes of potential interest for nuclear medicine research from various targets and ion sources. Based on the established yields, the activity accumulated over a $24 \mathrm{~h}$ implantation period was extrapolated. The data shows that in particular for some lanthanides very high activities can be collected. However, for relatively long-lived isotopes, such as ${ }^{225} \mathrm{Ra}$ and ${ }^{225} \mathrm{Ac}$ larger activities could be obtained by simply increasing the implantation period.

TRIUMF receives federal funding via a contribution agreement through the National Research Council of Canada. We also acknowledge additional support through Discovery Grants from the Natural Sciences and Engineering Research Council of Canada (NSERC): SAPIN/00021/2014 (P. Kunz) and SAPIN/00039/2017 (J. Lassen). Thanks to the ISAC operations crew for their support

\section{References}

[1] M. Dombsky, P. Kunz, Hyperfine Interactions 225, 17 (2013)

[2] J. Dilling, ISAC and ARIEL: The TRIUMF radioactive beam facilities and the scientific program 
(Springer, New York, 2014), ISBN 978-94-0077962-4

[3] P. Kunz, ISAC Yield Database https://mis.triumf.ca/science/planning/yield/beam (2018), https://mis.triumf.ca/science/ planning/yield/beam

[4] A.K.H. Robertson, C.F. Ramogida, P. Schaffer, V. Radchenko, Current Radiopharmaceuticals 11, 156 (2018)

[5] P. Bricault, M. Dombsky, A. Dowling, M. Lane, Nuclear Instruments and Methods in Physics Research Section B: Beam Interactions with Materials and Atoms 204, 319 (2003)

[6] M. Dombsky, P. Bricault, V. Hanemaayer, Nucl. Phys. A 746, 32c (2004)

[7] P. Kunz, P. Bricault, M. Dombsky, N. Erdmann, V. Hanemaayer, J. Wong, K. Lützenkirchen, Journal of Nuclear Materials 440, 110 (2013)

[8] V. Hanemaayer, P. Bricault, M. Dombsky, Nuclear Instruments and Methods in Physics Research Section B: Beam Interactions with Materials and Atoms 266, 4334 (2008)

[9] P.G. Bricault, F. Ames, M. Dombsky, P. Kunz, J. Lassen, A. Mjøs, J. Wong, Nuclear Instruments and Methods in Physics Research Section B: Beam Interactions with Materials and Atoms 366, 34 (2016)
[10] J.F. Ziegler, M. Ziegler, J. Biersack, Nuclear Instruments and Methods in Physics Research Section B: Beam Interactions with Materials and Atoms 268, 1818 (2010)

[11] J. Allison, K. Amako, J. Apostolakis, P. Arce, M. Asai, T. Aso, E. Bagli, A. Bagulya, S. Banerjee, G. Barrand et al., Nuclear Instruments and Methods in Physics Research Section A: Accelerators, Spectrometers, Detectors and Associated Equipment 835, 186 (2016)

[12] E.P. Horwitz, M.L. Dietz, R. Chiarizia, Journal of Radioanalytical and Nuclear Chemistry Articles 161, 575 (1992)

[13] A.K.H. Robertson, C.F. Ramogida, C. RodríguezRodríguez, S. Blinder, P. Kunz, V. Sossi, P. Schaffer, Physics in Medicine and Biology 62, 4406 (2017)

[14] N.A. Thiele, V. Brown, J.M. Kelly, A. AmorCoarasa, U. Jermilova, S.N. MacMillan, A. Nikolopoulou, S. Ponnala, C.F. Ramogida, A.K.H. Robertson et al., Angewandte Chemie International Edition 56, 14712 (2017)

[15] J.R. Crawford, P. Kunz, H. Yang, P. Schaffer, T.J. Ruth, Applied Radiation and Isotopes 122, 222 (2017)

[16] J.R. Crawford, H. Yang, P. Kunz, D.S. Wilbur, P. Schaffer, T.J. Ruth, Nuclear Medicine and Biology 48, 31 (2017) 